\title{
Use of prophylactic growth factors and antimicrobials in elderly patients with cancer: a review of the Medicare database
}

\author{
Romina Sosa ${ }^{1}$ - Shuling $\mathrm{Li}^{2}$ • Julia T. Molony ${ }^{2} \cdot$ Jiannong Liu $^{2}$ • Scott Stryker ${ }^{3}$. \\ Allan J. Collins ${ }^{2,4}$
}

Received: 7 October 2016 / Accepted: 17 April 2017 /Published online: 29 April 2017

(C) Springer-Verlag Berlin Heidelberg 2017

\begin{abstract}
Purpose Growth factors and antimicrobials can reduce complications of chemotherapy-induced myelosuppression. Their prophylactic use in elderly patients is important given the associated comorbidity in this age group. There is a developing trend by payers to include supportive care agents in chemotherapy care bundles, which could affect clinical practice. We examined whether the febrile neutropenia (FN) risk categories can be used to describe utilization in the Centers for Medicare \& Medicaid fee-for-service system in older adults. Methods We conducted a retrospective cohort study using the Medicare 20\% sample data to describe growth factor and antimicrobial use patterns in patients receiving chemotherapy for breast cancer, lung cancer, and non-Hodgkin lymphoma (NHL).

Results The highest percentage of patients receiving granulocyte colony-stimulating factor (GCSF) within the first 5 days of a chemotherapy cycle were on high-FN-risk regimens, particularly for cycle $1(73.7 \%$, breast cancer; $61.5 \%$, NHL) and cycle $2(75.9 \%$, breast cancer; $77.5 \%$, NHL). Chemotherapy
\end{abstract}

Electronic supplementary material The online version of this article (doi:10.1007/s00520-017-3720-x) contains supplementary material, which is available to authorized users.

Allan J. Collins

ACollins@cdrg.org

1 Baylor College of Medicine, Houston, TX, USA

2 Chronic Disease Research Group, Minneapolis Medical Research Foundation, 914 South 8th Street, Suite S4.100, Minneapolis, MN 55404, USA

3 Amgen Inc, Thousand Oaks, CA, USA

4 Department of Medicine, University of Minnesota, Minneapolis, MN, USA regimens for lung cancer are less myelotoxic, and growth factor use was more likely with latter cycles. Antibiotic use was lower at $15 \%$ within a cycle and appeared to be in response to complications.

Conclusion Practitioners use GCSF and antibiotics for elderly patients treated with potentially toxic chemotherapy, while comorbidity burden plays a role for patients treated with less myelotoxic regimens. The complexity of these choices in clinical practice should be considered in the proposed reimbursement changes being piloted by Medicare and private insurance companies seeking treatment cost reductions, as altered use could affect safety and efficacy.

Keywords Antimicrobials $\cdot$ Chemotherapy $\cdot$ Febrile neutropenia $\cdot$ Granulocyte colony-stimulating factor

\section{Introduction}

Febrile neutropenia (FN) is a well-known complication of myelosuppressive chemotherapy. Hospitalizations for FN often result in chemotherapy treatment delays or dose reductions in subsequent cycles, reducing the efficacy of chemotherapy and increasing diagnostic and treatment costs [1,2]. Use of supportive care agents has reduced complications from chemotherapy-induced myelosuppression. Primary prophylaxis with granulocyte colony-stimulating factor (GCSF), a biological agent that regulates the proliferation and survival of myeloid cells, successfully reduces the time of neutropenia and decreases FN incidence [3, 4]. Prophylactic antimicrobials, though not commonly used in the primary prophylaxis setting, reduce the number and severity of infectious episodes in patients with leukemia and lymphoma [5-7].

Several cancer consortiums, such as the National Comprehensive Cancer Network (NCCN), American 
Society of Clinical Oncology, and European Organization for Research and Treatment of Cancer, endorse the use of GCSFs and antibiotics to minimize adverse events associated with cancer therapy. In this study, we used NCCN guidelines to assign patients to one of three risk categories for development of FN and need for GCSF based on chemotherapy regimen and patient risk factors: high risk $(>20 \%)$, intermediate risk (10-20\%), and low risk $(<10 \%)$ [8]. Consensus recommendations center on significant reduction of $\mathrm{FN}$ following primary GCSF prophylaxis when the FN risk is $>20 \%$ [9]. Furthermore, some studies have demonstrated a significant reduction in FN with GCSF prophylaxis in patients with breast cancer receiving intermediate-risk chemotherapy when comorbidity increased the risk of infection [10]. Use of prophylactic antibiotics $[11,12]$ during chemotherapy is recommended in patients with anticipated neutropenia (absolute neutrophil count $<1000$ ) for longer than 7 days. Prophylactic antibiotic use is limited in solid tumor patients. Use of antivirals and antifungals is reserved for patients with prolonged neutropenia ( $>7-10$ days), typically experienced by patients with hematologic malignancies or undergoing stem cell transplant. Use of supportive care therapies has gained attention as the Centers for Medicare \& Medicaid Services (CMS) develops alternative payment models to contain the increasing cost of cancer treatments. Policy makers, insurers, and CMS have looked at alternative payment models. CMS is currently testing a 6-month chemotherapy period pilot payment model assessing total expenditures in a risk model (https:// innovation.cms.gov/initiatives/Oncology-Care/). Because supportive care agents are an expensive component of chemotherapy care, we investigated how these services are used in routine clinical care and if the proposed FN risk systems can adequately describe utilization in the CMS feefor-service system. We studied elderly patients treated for one of three cancer types: breast cancer, lung cancer, and nonHodgkin lymphoma (NHL).

\section{Methods}

We conducted a retrospective cohort study using the Medicare $20 \%$ sample data for all Medicare beneficiaries between January 1, 2007, and December 31, 2011. The database, maintained by CMS, includes the annual denominator file (information on demographics and enrollment in Medicare for each beneficiary) and the annual claims-based standard analytic files (SAFs). The SAFs contain Part A institutional, Part B physician/supplier, and Part D oral prescription drug claims. These claims-based files include information on diagnoses, procedures performed, chemotherapeutic and GCSF agents administered in outpatient settings, dates of service, and oral antimicrobials prescribed and filled.
Patients were eligible for the study if they started a chemotherapy course between July 1, 2007, and November 30, 2011, to treat breast cancer, lung cancer, or NHL at age 65 years or older; were continuously enrolled in Medicare Part A and Part B from 6 months before the start of the chemotherapy course to the last day of the course; and had Medicare Part D coverage from 30 days before the start of the course to the last day of the course or the sixth cycle end date, whichever came first. Patients were excluded who were enrolled in managed care organizations or who had any medical claims for stem cell transplant during the period from 6 months before the start of the chemotherapy course to the last day of the course.

Patients with breast cancer, lung cancer, or NHL were identified using International Classification of Diseases, Ninth Revision, Clinical Modification (ICD-9-CM) diagnosis codes (breast cancer, 174.xx, 175.xx; lung cancer, 162.xx-163.xx; NHL, 200.xx, 202.xx). Chemotherapy was identified using billing codes for chemotherapy administration and/or agents (Supplementary Table S1). Chemotherapy course and cycle were defined following the algorithm used by Choi et al. [13]. A chemotherapy course began at the first chemotherapy claim (index date) between January 1, 2007, and December 31,2011 , and ended at the last chemotherapy claim with a gap of less than 60 days between two consecutive claims. The first chemotherapy cycle was defined as beginning with the index date and ending with the next chemotherapy claim that occurred at least 20 days after the index date. Subsequent cycles were defined using similar rules up to a maximum of 6 cycles (including the first). For patients with two or more chemotherapy courses identified between July 1, 2007, and November 30,2011 , only the first was used in this study.

According to NCCN guidelines for myeloid growth factors use [8], we classified chemotherapy regimens as high $(>20 \%)$ and intermediate (10-20\%) FN risk based on the chemotherapeutic agents on the claims. Regimens not defined as high or intermediate $\mathrm{FN}$ risk were combined into an unclassified FN risk category. To further evaluate unclassified FN risk regimens, we identified the number of chemotherapeutic agents by level of myelosuppression (high, low, none) according to the definition from a prior study by Wecker and colleagues [14]. For each cycle, we categorized patients into one of the following four groups in descending hierarchical order: (1) two or more high myelosuppressive agents, (2) exactly one high myelosuppressive agent, (3) one or more low myelosuppressive agents in the absence of high myelosuppressive agents, and (4) none (mainly biologic agents, for example, rituximab). Patient-cycles were excluded if information on myelosuppression level was not available for every agent used in the cycle.

We defined comorbid conditions 6 months before the start of chemotherapy using one or more Medicare Part A inpatient, skilled nursing facility, or home health agency claims or two or more Medicare Part A outpatient or Part B claims at least 30 days apart with qualifying ICD-9-CM diagnosis codes 
(Supplementary Table S2). We also defined lengths of hospital stays and cause-specific hospitalizations, including cardiovascular disease, infectious disease, and other. The follow-up period was from the course start date to the earliest of the following: death; change in Medicare enrollment status for Parts A, B, and D; the last day of the sixth cycle; or the course end date.

For each cycle, we identified supportive treatment with GCSF and oral antimicrobial use. Administration of GCSF was identified using HCPCS codes (filgrastim: J1440 and J1441; pegfilgrastim: J2505; sargramostim: J2820) in Medicare Part A outpatient or Part B claims. Primary prophylactic use of GCSF was defined as first GCSF administered from cycle start date (day 0 ) to day 5 . Oral antimicrobial agents including antibacterials, antivirals, and antifungals were identified in Medicare Part D claims using National Drug Codes (Supplementary Table S3). Part D claims for oral medications represent the date of prescription fill, which may not indicate the date of first use. Thus, for prescription fills during 5 days before day 0 , use was considered to begin on day 0 for potential infection prophylaxis, and these fills were included in the definition of oral antimicrobials used during cycle days 0 through 5. For each patient, only the first prescription fill for any antimicrobial in each cycle was considered.

For each chemotherapy cycle, we illustrated supportive treatment with GCSF and oral antimicrobials from day 0 through day 5 of the cycle by chemotherapy regimen FN risk category. For patients receiving unclassified FN risk regimens, we further examined primary prophylactic use of GCSF by category of myelosuppression level of agents used within the cycle. For patients receiving GCSF anytime during the cycle, we described timing of GCSF initiation relative to the beginning of the cycle overall and by agent type for each day from day 0 through day 5 and over the entire period from day 6 to the end of the cycle. For patients with oral antimicrobial prescription fills anytime during the cycle, we examined timing of first prescription fill by each day during the period from 5 days before day 0 to the end of the cycle. Descriptive statistics (count and proportion) are reported. The analyses were repeated for each cycle up to 6 cycles for each cancer type. An exploratory analysis was performed to describe the type and duration of antimicrobial use for the first cycle of chemotherapy only. All analyses were performed using SAS, version 9.2 (SAS Institute Inc.).

\section{Results}

\section{Patient characteristics and chemotherapy regimens}

The study cohort included 9110 patients with breast cancer, 12,032 with lung cancer, and 6306 with NHL, treated with chemotherapy between July 1, 2007, and November 30, 2011. Most chemotherapy regimens were unclassified to FN risk: breast cancer, $80 \%$; lung cancer $57 \%$; NHL, $83 \%$. The proportion of patients on FN risk regimens classified as high and intermediate varied greatly across the three cancer types. Supplementary Table S4 summarizes the most commonly used regimens based on cancer type and $\mathrm{FN}$ risk classification.

Patient characteristics at baseline varied by cancer type and chemotherapy regimen FN risk category (Table 1). Breast cancer patients on high $\mathrm{FN}$ risk regimens were younger (82\% aged 65-74 years) and less likely to have comorbid conditions, prior infectious hospitalization, or long hospital stays ( $\geq 4$ days). NHL patients on high FN risk were younger (48 vs. $41 \%$ aged $65-74$ years) and less likely to have congestive heart failure or long hospital stays. Differences in lung cancer patients by regimen category were less profound.

\section{Supportive treatment utilization in first 5 days of chemotherapy cycle}

Figure 1 shows the patterns of GCSF and oral antimicrobial use in the first 5 days of a chemotherapy cycle by regimen FN risk category for each tumor type and chemotherapy cycle. In general, the highest percentage of patients receiving GCSF within the first 5 days of a chemotherapy cycle were on high FN risk regimens, particularly for cycle $1(73.7 \%$, breast cancer; $61.5 \%$, NHL) and cycle $2(75.9 \%$, breast cancer; $77.5 \%$, NHL). During the subsequent cycles, GCSF use steadily decreased to $34.0 \%$ in cycle 6 for breast cancer but remained stable at about $80 \%$ for NHL. In general, patients on intermediate FN risk regimens were less likely to receive GCSF; however, we noted an interesting increased pattern of use for lung cancer patients: $20 \%$ in cycle 1 to $40 \%$ in the last 3 cycles. A substantial proportion of patients on unclassified FN risk regimens received GCSF: approximately 20-40\% for cycles 3-6 for all three tumors. Compared with GCSF use, oral antimicrobial agent use from day 0 to day 5 of a chemotherapy cycle was considerably lower $(<15 \%)$, regardless of cancer type and regimen $\mathrm{FN}$ risk category.

Among patients treated with unclassified $\mathrm{FN}$ risk regimens, use of myelosuppressive agents for breast cancer, lung cancer, and NHL occurred in 93.1, 96.8, and $56.9 \%$ of total patientcycles, respectively. Primary prophylactic GCSF use was minimal in patients who received no myelosuppressive agents or low myelosuppressive agents only but greatly increased in patients who received high myelosuppressive agents, particularly those receiving two or more (Table 2).

\section{Timing of supportive treatment utilization}

Among all patients treated with chemotherapy, 24 to $54 \%$ received GCSF, depending on cancer type and cycle (Table 3). Among patients who received GCSF anytime during the first cycle, the most common GCSF agent used was pegfilgrastim (74-84\%), followed by filgrastim (14-23\%) and 
Table 1 Baseline patient characteristics, by cancer type and febrile neutropenia risk category of chemotherapy regimen

\begin{tabular}{|c|c|c|c|c|c|c|c|c|c|}
\hline \multirow[b]{3}{*}{ Patient characteristics } & \multicolumn{3}{|c|}{ Breast cancer $(n=9110)$} & \multicolumn{3}{|c|}{ Lung cancer $(n=12,032)$} & \multicolumn{3}{|c|}{ Non-Hodgkin lymphoma $(n=6306)$} \\
\hline & \multicolumn{3}{|c|}{ Risk of FN } & \multicolumn{3}{|c|}{ Risk of FN } & \multicolumn{3}{|c|}{ Risk of FN } \\
\hline & High & Intermediate & Unclassified & High & Intermediate & Unclassified & High & Intermediate & Unclassified \\
\hline Total patients & 923 & 908 & 7279 & 37 & 5130 & 6865 & 1035 & 24 & 5247 \\
\hline \multicolumn{10}{|l|}{ Age, years } \\
\hline $65-69$ & 48.1 & 27.6 & 28.9 & 48.6 & 26.7 & 26.1 & 19.9 & \multirow{2}{*}{$*$} & 20.7 \\
\hline $70-74$ & 33.8 & 28.6 & 28.9 & \multirow{2}{*}{$\begin{array}{l}32.4 \\
*\end{array}$} & 32.7 & 29.6 & 27.7 & & 20.5 \\
\hline $75-80$ & 14.4 & 21.9 & 20.0 & & 23.9 & 22.7 & 24.9 & \multirow{2}{*}{$\begin{array}{l}45.8 \\
*\end{array}$} & 20.9 \\
\hline$\geq 80$ & 3.7 & 21.8 & 22.2 & * & 16.7 & 21.6 & 27.4 & & 38.0 \\
\hline \multicolumn{10}{|l|}{ Race } \\
\hline White & 85.6 & 84.3 & 87.3 & \multirow{2}{*}{$\begin{array}{l}91.9 \\
*\end{array}$} & 88.9 & 87.3 & 89.6 & \multirow{3}{*}{$\begin{array}{l}91.7 \\
* \\
*\end{array}$} & 92.1 \\
\hline African American & 9.5 & 11.1 & 8.5 & & 7.5 & 7.8 & 4.3 & & 3.8 \\
\hline Other & 4.9 & 4.6 & 4.1 & $*$ & 3.5 & 4.9 & 6.1 & & 4.1 \\
\hline \multicolumn{10}{|l|}{ Sex } \\
\hline Male & 1.6 & $*$ & 1.0 & 40.5 & 48.2 & 48.0 & 43.3 & 54.2 & 42.3 \\
\hline Female & 98.4 & 98.9 & 99.0 & 59.5 & 51.8 & 52.0 & 56.7 & 45.8 & 57.7 \\
\hline \multicolumn{10}{|l|}{ Comorbid conditions $^{\mathrm{a}}$} \\
\hline ASHD & 6.3 & 12.2 & 12.7 & $*$ & 27.4 & 24.8 & 19.2 & $*$ & 18.3 \\
\hline $\mathrm{CHF}$ & 2.2 & 6.7 & 6.7 & \multirow[t]{2}{*}{$*$} & 9.4 & 10.0 & 6.4 & $*$ & 10.3 \\
\hline CVA/TIA & 2.0 & 4.0 & 3.3 & & 7.1 & 5.6 & 4.8 & $*$ & 4.4 \\
\hline Dysrhythmia & 5.7 & 13.0 & 11.3 & $*$ & 18.1 & 19.1 & 15.3 & $*$ & 16.9 \\
\hline PVD & 5.1 & 7.7 & 8.1 & \multirow{2}{*}{ * } & 15.1 & 14.7 & 11.0 & \multirow{2}{*}{$*$} & 10.3 \\
\hline Other CVD & 6.8 & 7.7 & 8.2 & & 9.9 & 10.6 & 11.7 & & 9.5 \\
\hline Anemia & 7.4 & 14.8 & 14.8 & \multirow[t]{2}{*}{35.1} & 18.2 & 20.5 & 25.0 & * & 26.5 \\
\hline CKD & 2.9 & 6.8 & 6.3 & & 9.2 & 8.9 & 11.9 & * & 11.7 \\
\hline COPD & 10.0 & 12.1 & 13.1 & 32.4 & 55.0 & 47.2 & 12.3 & * & 11.0 \\
\hline Diabetes & 21.6 & 23.1 & 23.6 & $*$ & 23.6 & 22.6 & 25.1 & $*$ & 21.6 \\
\hline Osteoarthritis & 10.8 & 10.7 & 11.4 & & 10.4 & 10.4 & 12.1 & $*$ & 9.8 \\
\hline Thyroid disease & 13.9 & 16.2 & 15.8 & $*$ & 11.3 & 11.8 & 14.8 & & 11.4 \\
\hline Other cancer & 1.7 & 6.5 & 8.9 & * & 7.0 & 8.9 & 11.6 & $*$ & 16.7 \\
\hline Primary cause of hosp & alization & & & & & & & & \\
\hline Infectious disease & 1.6 & 4.8 & 4.0 & * & 7.0 & 8.2 & 5.0 & $*$ & 5.3 \\
\hline CVD & $*$ & 3.9 & 3.9 & & 7.7 & 7.1 & 4.8 & $*$ & 5.6 \\
\hline Other & 37.2 & 34.4 & 30.6 & * & 41.0 & 36.8 & 34.4 & * & 21.0 \\
\hline Hospitalization LOS, & $\mathrm{ys}^{\mathrm{a}}$ & & & & & & & & \\
\hline 0 & 61.1 & 61.7 & 65.1 & 70.3 & 50.5 & 54.8 & 60.7 & 58.3 & 72.3 \\
\hline $1-3$ & 28.6 & 18.8 & 15.6 & $*$ & 8.5 & 8.1 & 7.8 & $*$ & 5.7 \\
\hline 4-6 & 6.7 & 9.3 & 8.7 & * & 14.5 & 12.8 & 9.9 & * & 7.6 \\
\hline$\geq 7$ & 3.6 & 10.2 & 10.5 & $*$ & 26.4 & 24.2 & 21.6 & $*$ & 14.4 \\
\hline
\end{tabular}

$A S H D$ atherosclerotic heart disease, $C H F$ congestive heart failure, $C K D$ chronic kidney disease, $C O P D$ chronic obstructive pulmonary disease, $C V A / T I A$ cerebrovascular accident/transient ischemic attack, $C V D$ cardiovascular disease, FR febrile neutropenia, GI gastrointestinal, $L O S$ length of stay, $P V D$ peripheral vascular disease

${ }^{\text {a }}$ Defined based on 6 months prior to chemotherapy initiation

${ }^{\mathrm{b}}$ Values for cells with ten or fewer patients are suppressed 

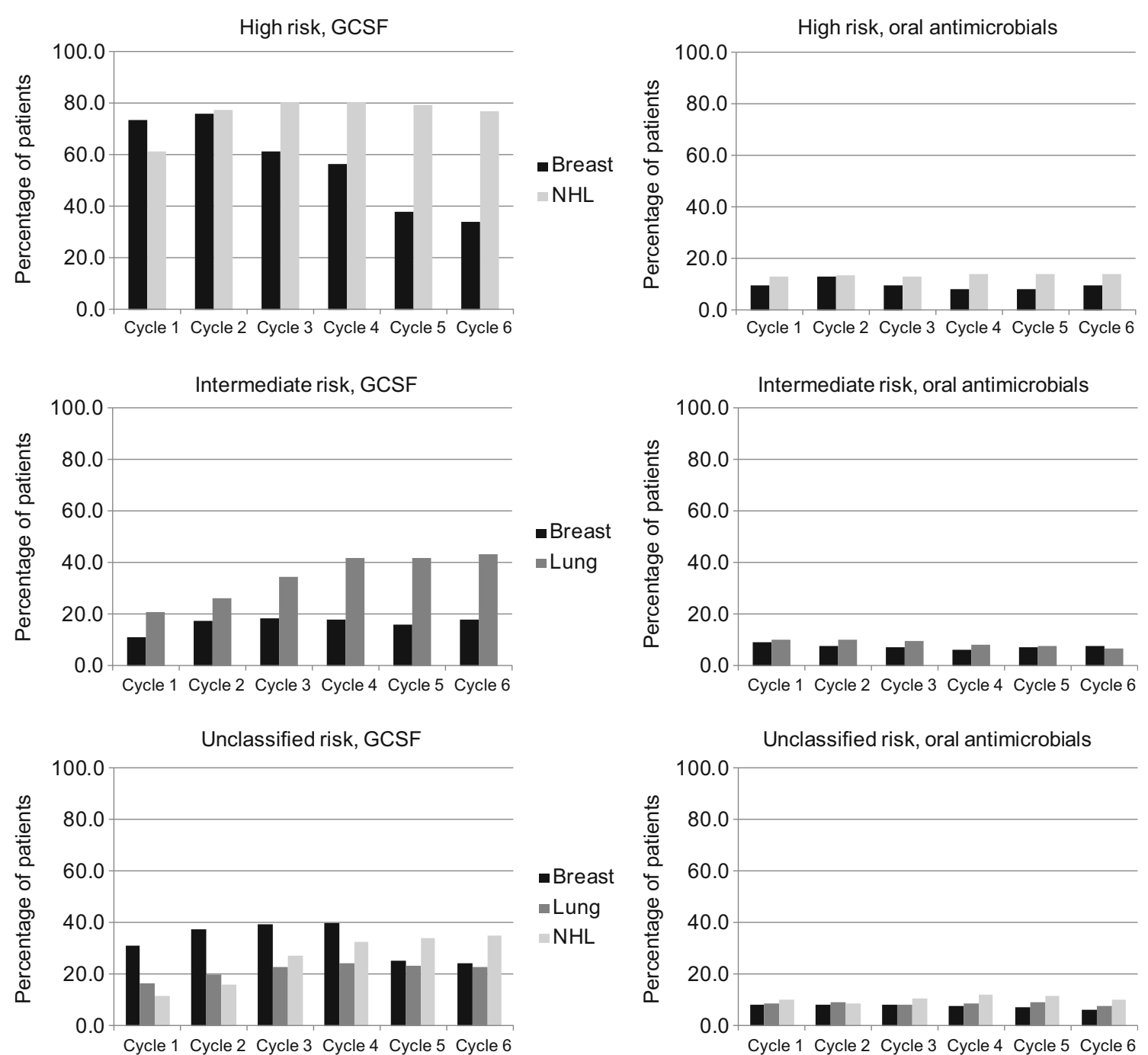

Fig. 1 Percentage of patients receiving a supportive treatment during days 0 to 5 of the chemotherapy cycle, by febrile neutropenia risk category of chemotherapy regimen and treatment type. GCSF granulocyte colony-stimulating factor, $N H L$ non-Hodgkin lymphoma

sargramostim (3-4\%). During the first cycle, timing of any GCSF use relative to the cycle start date was varied. For breast cancer and NHL, GCSF use peaked on the day after the beginning of the cycle (breast cancer, $66.2 \%$; NHL, 39.5\%) and greatly decreased from day 2 (breast cancer, $5.9 \%$; NHL, $17.6 \%$ ) to day 5 (breast cancer, $0.6 \%$; NHL, $3.1 \%$ ). For lung cancer, however, GCSF use peaked on day $1(30.9 \%)$ and day $3(19.2 \%)$. About 3 to $6 \%$ of GCSF was given on the cycle start date. More pegfilgrastim was given on days 0 through 5 and more filgrastim after day 5 . Use patterns were similar by agent type and for the subsequent cycles.

About 20 to $31 \%$ of patients had at least one prescription fill for an oral antimicrobial agent during the chemotherapy cycle, depending on cancer type and cycle (Table 3). For breast cancer patients, the first prescription fill during the first cycle occurred in the 5 days before the start of the first cycle for $13.9 \%$ of patients; days $0-5,17.6 \%$; and after day $5,68.6 \%$. Peak days for first prescription fill were days 0,7 , and 14 of the first chemotherapy cycle (Fig. 2). Similar patterns occurred for subsequent cycles and for patients with lung cancer and NHL.

Of the overall prescriptions for antimicrobials filled during the first cycle, most were for antibacterial agents; $77 \%$ of patients with NHL and $86 \%$ of patients with breast cancer filled antibacterial agents. Antifungals were the second most filled antimicrobial for breast cancer (10\%) and lung cancer (11\%) patients, and antivirals were the second most filled antimicrobial for NHL patients (13\%). Median length of antimicrobial treatment during the first cycle of chemotherapy was 1 week for antibacterials and antifungals across all cancer types.

\section{Discussion}

In this study, we used the Medicare database to assess use of supportive care agents, specifically GCSF and antimicrobials, 
Table 2 Percentage of patients receiving prophylactic GCSF by chemotherapy cycle and myelosuppression level of agents used in the cycle among patients who were treated with regimens not classified as high or intermediate risk of febrile neutropenia based on the NCCN guideline

\begin{tabular}{|c|c|c|c|c|c|c|c|c|}
\hline \multirow[b]{3}{*}{ Cancer Type and Cycle } & \multicolumn{8}{|c|}{ Myelosuppression Level } \\
\hline & \multicolumn{2}{|l|}{ None } & \multicolumn{2}{|c|}{$\geq 1$ Low, No High } & \multicolumn{2}{|c|}{1 High } & \multicolumn{2}{|c|}{$\geq 2$ High } \\
\hline & $n$ & $\%$ & $n$ & $\%$ & $n$ & $\%$ & $n$ & $\%$ \\
\hline \multicolumn{9}{|l|}{ Breast cancer } \\
\hline 1 & 870 & \multirow{3}{*}{ * } & 705 & $*$ & 1545 & 4.8 & 3798 & 56.8 \\
\hline 2 & 465 & & 633 & 3.6 & 1366 & 6.2 & 3592 & 62.2 \\
\hline 3 & 296 & & 618 & 2.3 & 1316 & 13.9 & 3185 & 62.4 \\
\hline 4 & 168 & & 593 & 2.9 & 1260 & 17.1 & 2738 & 62.9 \\
\hline 5 & 125 & \multirow[b]{2}{*}{ * } & 686 & 3.5 & 1183 & 16.8 & 1200 & 53.7 \\
\hline 6 & 96 & & 726 & 2.3 & 971 & 14.9 & 983 & 55.6 \\
\hline \multicolumn{9}{|l|}{ Lung cancer } \\
\hline 1 & 309 & \multirow{2}{*}{$\begin{array}{l}* \\
*\end{array}$} & 580 & $*$ & 2323 & 7.4 & 3435 & 26.4 \\
\hline 2 & 182 & & 487 & 5.1 & 2164 & 11.0 & 3213 & 29.9 \\
\hline 3 & 121 & $*$ & 433 & 7.2 & 1914 & 12.5 & 2693 & 33.6 \\
\hline 4 & 83 & & 376 & 8.0 & 1624 & 14.2 & 2198 & 35.9 \\
\hline 5 & 102 & & 299 & 7.7 & 1348 & 16.6 & 1363 & 36.7 \\
\hline 6 & 105 & & 261 & 8.8 & 1161 & 15.8 & 1018 & 39.1 \\
\hline \multicolumn{9}{|l|}{ Non-Hodgkin lymphoma } \\
\hline 1 & 2941 & 0.9 & 113 & $*$ & 1350 & 22.8 & 480 & 45.0 \\
\hline 2 & 2368 & 1.0 & 85 & * & 1321 & 29.9 & 494 & 51.4 \\
\hline 3 & 660 & 2.4 & 70 & $*$ & 1195 & 32.5 & 428 & 57.9 \\
\hline 4 & 350 & 4.6 & 61 & 19.7 & 1011 & 36.1 & 343 & 61.8 \\
\hline 5 & 263 & 5.7 & 53 & 24.5 & 796 & 37.6 & 254 & 64.2 \\
\hline 6 & 181 & $*$ & 40 & $*$ & 632 & 39.9 & 206 & 63.6 \\
\hline
\end{tabular}

Patient-cycles were excluded if information on myelosuppression level was not available for every agent used in the cycle

GCSF granulocyte colony-stimulating factor, NCCN National Comprehensive Cancer Network

${ }^{\text {a }}$ Values for cells with ten or fewer patients are suppressed associated with cancer therapy in the elderly population. We explored utilization patterns of GCSF and antibiotics to help understand how these supportive care agents may be affected under a new CMS payment system (https://innovation.cms. gov/initiatives/Oncology-Care/).

We searched the Medicare 20\% sample database for regimens associated with high, intermediate, and low risk for FN, as determined by NCCN guidelines. We found that patients with breast cancer or NHL were more frequently treated with high-risk regimens than were lung cancer patients, who primarily received intermediate-risk regimens. As anticipated, most GCSF use occurred in patients treated with high-risk regimens, with more than $60 \%$ of breast cancer patients and $80 \%$ of NHL patients receiving GCSF support at any given therapy cycle. Interestingly, while GCSF use remained constant through all cycles in NHL patients, use in breast cancer patients declined after cycle 2, a possible extrapolation from clinical trials in breast cancer $[10,15,16]$ that observed the highest risk of FN occurring during the first 2 cycles of chemotherapy. Recent data suggest that primary GCSF use throughout all chemotherapy cycles minimizes episodes of FN among patients with early breast cancer treated with high- and intermediate-risk regimens [17]. An analysis of subsequent years would be informative to determine how practitioners are applying the new evidence to supportive care.

In lung cancer patients, our data demonstrate greater use of GCSF with later cycles of chemotherapy. These patients have several comorbid conditions, such as coronary artery disease and chronic obstructive pulmonary disease, possibly associated with cigarette use (Table 1) [18]. Increasing GCSF use as cycles progress suggests a pattern in which complications early in the treatment course lead to increased supportive care measures in subsequent cycles to prevent complications and treatment delays. Our analysis demonstrates that in addition to chemotherapy-associated risk of FN, physicians also use patient risk factors to determine the need for growth factor support. Recent studies suggest that chemotherapy risk factor alone is not sufficient to predict the need for growth factor support [19, 20], and that a history of chronic comorbid conditions increases the risk of FN [19]. Thus far, the NCCN guidelines have 
Table 3 GCSF use during a chemotherapy cycle and timing of GCSF relative to the beginning of the cycle for selected cycles, by cancer type

\begin{tabular}{|c|c|c|c|c|c|c|c|c|c|}
\hline & \multicolumn{3}{|l|}{ Breast cancer } & \multicolumn{3}{|l|}{ Lung cancer } & \multicolumn{3}{|c|}{ Non-Hodgkin lymphoma } \\
\hline & $\begin{array}{l}\text { Cycle } 1 \\
(n=9110)\end{array}$ & $\begin{array}{l}\text { Cycle 3 } \\
(n=7275)\end{array}$ & $\begin{array}{l}\text { Cycle } 5 \\
(n=4691)\end{array}$ & $\begin{array}{l}\text { Cycle } 1 \\
(n=12,032)\end{array}$ & $\begin{array}{l}\text { Cycle } 3 \\
(n=8874)\end{array}$ & $\begin{array}{l}\text { Cycle } 5 \\
(n=4712)\end{array}$ & $\begin{array}{l}\text { Cycle } 1 \\
(n=6306)\end{array}$ & $\begin{array}{l}\text { Cycle } 3 \\
(n=3497)\end{array}$ & $\begin{array}{l}\text { Cycle } 5 \\
(n=2226)\end{array}$ \\
\hline $\begin{array}{l}\text { GCSF use during the } \\
\text { cycle, } n \text { (\% total) }\end{array}$ & $3591(39.4)$ & $3275(45.0)$ & 1489 (31.7) & $3223(26.8)$ & $3148(35.5)$ & $1721(36.5)$ & $1535(24.3)$ & $1644(47.0)$ & $1196(53.7)$ \\
\hline \multicolumn{10}{|c|}{ GCSF use by agent type, $n(\%)$} \\
\hline Pegfilgrastim & $3000(83.5)$ & $2734(83.5)$ & $1143(76.8)$ & $2372(73.6)$ & $2341(74.4)$ & $1242(72.2)$ & $1278(83.3)$ & $1424(86.6)$ & $1041(86.6)$ \\
\hline Filgrastim & $484(13.8)$ & $470(14.4)$ & $301(20.2)$ & $732(22.7)$ & $690(21.9)$ & 407 (23.7) & $218(14.2)$ & $182(11.1)$ & $128(10.7)$ \\
\hline Sargramostim & $97(2.7)$ & $71(2.2)$ & $45(3.0)$ & $119(3.7)$ & $117(3.7)$ & $72(4.2)$ & $39(2.5)$ & $38(2.3)$ & $27(2.3)$ \\
\hline \multicolumn{10}{|c|}{ GCSF use, by cycle day (\%) } \\
\hline 0 (cycle start date) & 6.2 & 7.4 & 6.9 & 3.4 & 4.6 & 4.7 & 5.1 & 5.5 & 6.9 \\
\hline 1 & 66.2 & 68.9 & 62.1 & 30.9 & 35.1 & 36.9 & 39.5 & 52.5 & 53.7 \\
\hline 2 & 5.9 & 5.7 & 6.0 & 7.0 & 7.7 & 8.2 & 17.6 & 16.2 & 17.5 \\
\hline 3 & 4.4 & 4.0 & 5.0 & 19.2 & 23.0 & 22.7 & 10.4 & 9.4 & 8.7 \\
\hline 4 & 0.9 & 1.3 & 1.3 & 2.9 & 3.1 & 2.9 & 5.7 & 3.3 & 2.8 \\
\hline 5 & 0.6 & 1.0 & 1.0 & 4.1 & 3.3 & 3.7 & 3.1 & 2.4 & 1.5 \\
\hline 6 to cycle end date & 15.8 & 11.7 & 17.6 & 32.5 & 23.2 & 20.9 & 18.7 & 10.8 & 8.9 \\
\hline \multicolumn{10}{|c|}{ Pegfilgrastim use, by cycle day (\%) } \\
\hline 0 & 7.1 & 8.0 & 7.3 & 4.4 & 4.8 & 5.4 & 4.9 & 5.3 & 6.4 \\
\hline 1 & 74.9 & 75.6 & 69.8 & 37.9 & 38.6 & 40.4 & 44.6 & 56.3 & 57.5 \\
\hline 2 & 5.9 & 5.6 & 6.4 & 8.5 & 8.3 & 8.9 & 19.0 & 16.8 & 18.0 \\
\hline 3 & 4.6 & 3.9 & 4.2 & 24.2 & 27.1 & 27.0 & 11.9 & 10.1 & 9.3 \\
\hline 4 & 0.7 & 0.9 & 1.0 & 3.0 & 3.0 & 3.1 & 5.3 & 2.7 & 2.9 \\
\hline 5 & 0.4 & 0.4 & * & 4.6 & 3.5 & 3.7 & 3.0 & 2.1 & 1.4 \\
\hline 6 to cycle end date & 6.4 & 5.6 & 10.9 & 17.5 & 14.7 & 11.4 & 11.3 & 6.8 & 4.4 \\
\hline \multicolumn{10}{|c|}{ Filgrastim use, by cycle day (\%) } \\
\hline 0 & $*$ & 4.5 & 6.0 & $*$ & 4.2 & 2.9 & 6.0 & $*$ & 9.4 \\
\hline 1 & 19.4 & 32.8 & 36.9 & 11.2 & 24.8 & 28.3 & 11.5 & 28.6 & 29.7 \\
\hline 2 & 5.1 & 6.0 & 4.3 & 2.5 & 5.8 & 6.4 & 8.3 & 8.8 & 12.5 \\
\hline 3 & 2.8 & 4.3 & 7.6 & 4.8 & 10.4 & 10.6 & * & 6.0 & * \\
\hline 4 & $*$ & 3.6 & $*$ & 2.3 & 3.5 & $*$ & 7.3 & 7.7 & $*$ \\
\hline 5 & $*$ & 4.0 & * & 2.7 & 2.8 & 3.4 & * & * & * \\
\hline 6 to cycle end date & 68.2 & 44.9 & 39.5 & 76.0 & 48.6 & 45.9 & 60.1 & 40.1 & 39.1 \\
\hline $\begin{array}{l}\text { Prescription filled for } \\
\text { oral antimicrobial } \\
\text { agent during the } \\
\text { cycle, } n(\% \text { total })\end{array}$ & $2378(26.1)$ & $1611(22.1)$ & $940(20.0)$ & $3398(25.5)$ & $2231(25.1)$ & $1127(23.9)$ & $1663(26.4)$ & $1042(29.8)$ & $688(30.9)$ \\
\hline \multicolumn{10}{|c|}{ Prescription filled, by cycle day $(\%)$} \\
\hline 5 days before day 0 & 13.9 & 14.5 & 14.2 & 14.4 & 14.5 & 14.1 & 17.3 & 12.9 & 15 \\
\hline Day 0 to day 5 & 17.6 & 22.5 & 21.6 & 18.1 & 20.6 & 21.7 & 22.8 & 25.1 & 24.7 \\
\hline $\begin{array}{l}\text { Day } 6 \text { to the end of } \\
\text { the cycle }\end{array}$ & 68.5 & 62.9 & 64.3 & 67.5 & 65.0 & 64.2 & 59.9 & 62.0 & 60.3 \\
\hline
\end{tabular}

${ }^{\text {a }}$ Values for cells with ten or fewer patients are suppressed

endorsed clinical latitude to practitioners in prescribing GCSF to patients in clinical scenarios outside of chemotherapy-associated risk. This will most likely create challenges under a bundling system, as there is no algorithm to predict necessity using comorbid conditions. These results support the need for monitoring systems that address safety in anticipated changes in practice, with an emphasis on the efficacy of ancillary care interventions to prevent infectious complications.

Use of prophylactic antimicrobials is not currently recommended for solid tumors [21]. Consistent with this recommendation, we found that prophylactic antimicrobial use was less frequent than GCSF use (Table 3 and Fig. 1). Overall, 
Fig. 2 Percentage of patients with first oral antimicrobial prescription filled during the chemotherapy cycle by day of cycle for each cancer type
Breast Cancer

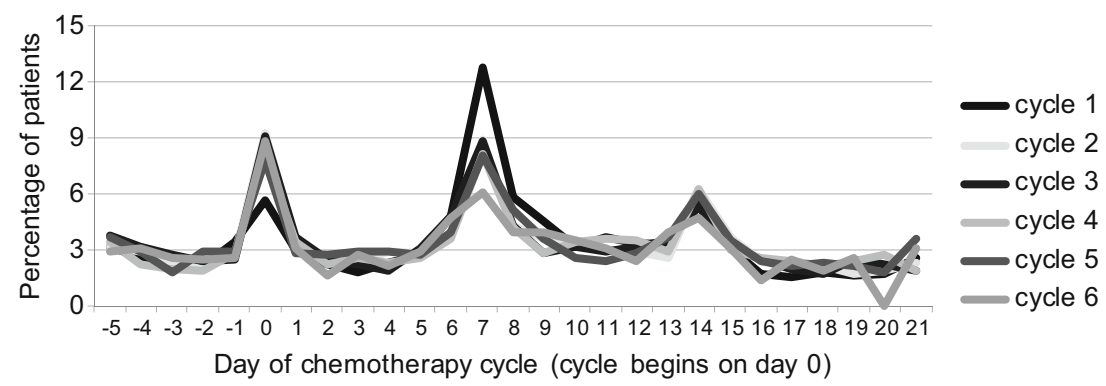

Lung Cancer

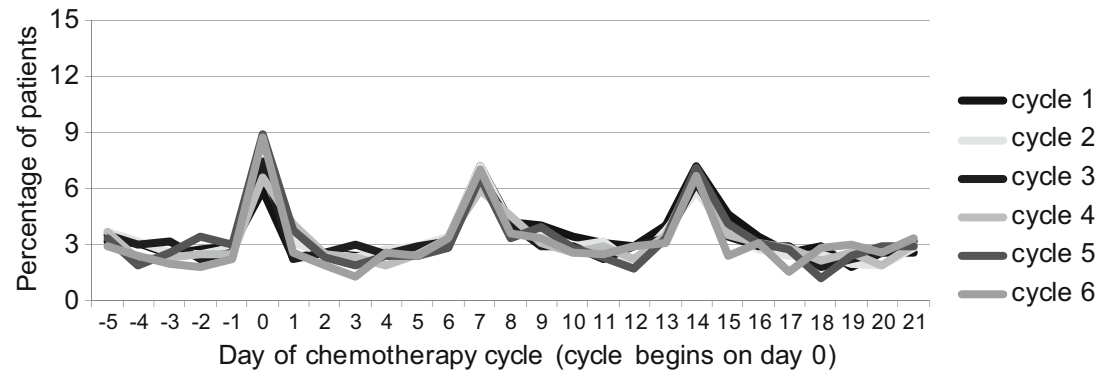

Non-Hodgkin Lymphoma

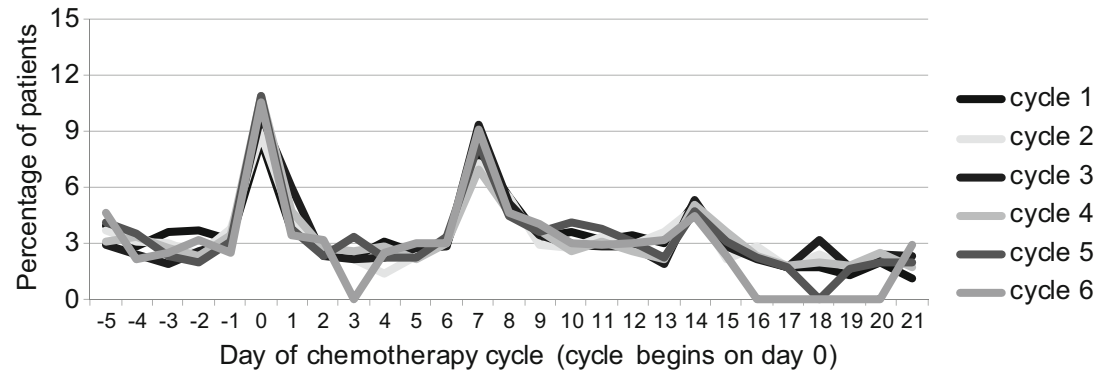

antimicrobials were used only $10-15 \%$ of the time across different cancer types. Prophylactic antimicrobials were more likely to be used in patients with NHL treated with highly myelosuppressive regimens, as expected due to the greater potential for neutropenia associated with NHL regimens [22]. Antimicrobial prescription data revealed two distinct peaks across cycles: at day 0 and day 7 (Fig. 2) for all cancer types. These could reflect providers' anticipated or actual treatment for infection during the myelosuppressive period. Day 0 may reflect prophylactic use of antimicrobials or physicians providing antibiotic prescription in anticipation of potential febrile complications; day 7 likely represents empiric use for a febrile episode consistent with expected neutropenia 7-10 days after chemotherapy administration. Our interpretation is limited as we were able to gather information only on fill dates and not on actual compliance with oral administration. However, given the low frequency of use, this is unlikely to affect our interpretation of the data.

The greatest strength of this study is that it provides a realistic representation of supportive care drug use in the elderly population with cancer in the USA, as most patients in this country aged older than 65 years are covered by Medicare, making our database a reliable gage of practice patterns in the elderly population. Our intent was to describe utilization in the CMS fee-for-service system of the use of growth factors and antibiotics based on a risk stratification system that utilized chemotherapy risk as defined by NCCN [8]. An anticipated limitation is that most chemotherapy regimens used in the Medicare population are not included in the examples provided by the NCCN and had to be labeled as "unclassified" in our study. We compensated for this by using a sensitivity analysis to examine whether the drug combinations in the unclassified risk category included highly myelosuppressive agents. We employed a method previously described by Weycker et al. [14]. By using this method, we found that in both breast and lung cancer, several patients in the unclassified risk category receiving GCSF were treated with one or more agents considered highly myelosuppressive (Supplementary Table S4), thus providing justification for the use of growth factor support.

An important extrapolation of our data revealed that in addition to chemotherapy myelosuppressive risk, practitioners integrate patient comorbid conditions in their assessment of 
FN risk and need for GCSF administration. The guidelines do not provide a clear formula for how to predict the risk of neutropenia based on patient comorbidity and leave this to physician discretion. Clearly, comorbid conditions, tumorrelated characteristics, and the combination of agents and dosing all play important roles in FN risk. Studies attempting to correlate the risk of FN as assessed by physicians when compared with a validated multivariate model revealed a weak association, supporting the need to develop algorithms incorporating patient characteristics that reliably predict benefit of supportive care agents [23].

Our assessment of provider practice patterns for supportive care data assessment should inform physicians under the new CMS-proposed payment model that diversity of treatment is complex. A simple risk model relying on NCCN chemotherapy risk guidelines is not sufficient. The Oncology Care Model (OCM), a new payment model launched on July 1, 2016 , requires and pays for care coordination at $\$ 160 /$ month for participating patients based on a 6-month period of cancer chemotherapy and care coordination. Total services are assessed from all Medicare Part A, B, and D claims retrospectively during the chemotherapy-defined period of care. This model leaves the existing service billing system intact but assesses the total care expenditures billed. The proposed CMS total savings approach lets providers select the various care delivery options over a longer time period. Performance measures discussed by CMS include supportive and palliative care options. Interestingly, specifics related to hospitalization for infection were lacking. This may be important since FN and hospitalizations for infection are among the costliest complications of cancer therapy. Growth factor support is an effective yet costly therapy that reduces FN complications. Oral prophylactic antibiotic use is less costly but whether it has equivalent benefit to growth factor support is unclear, especially regarding solid tumors in the elderly population.

In conclusion, use of GCSF and antibiotics during chemotherapy for breast, lung, and NHL cancers is complex and not easily determined in a simple risk model that uses only chemotherapy-associated FN risk. Our data demonstrate that supportive care is individualized in the elderly/Medicare population based on the diverse comorbidity burden and intensity of chemotherapy. Unfortunately, there are no existing algorithms to predict benefit based on this combination of chemotherapy and patient risk factors; hence, we rely on physician discretion to anticipate supportive care needs. This may prove to be challenging under the proposed CMS OCM, as there are no models to help us predict clinical outcomes of FN based on both chemotherapy intensity and patient comorbidity. There may be a tendency to either withhold supportive care drugs to minimize immediate costs or to overprescribe these medications to avoid complications of hospitalization. The period-ofcare payment system continues to allow providers to make therapy choices while assessing total costs. Ongoing assessments of the new payment system will be important to determine whether physician practices change under the new OCM and whether patient outcomes are comparable or better than under the traditional fee-for-service system, particularly related to infection complications.

Acknowledgements The authors thank Minneapolis Medical Research Foundation colleague Nan Booth, MSW, MPH, ELS, for the manuscript editing.

\section{Compliance with ethical standards}

Conflict of interest Dr. Sosa reports no conflicts of interest. Drs. Li and Liu and Ms. Molony are employed by the Chronic Disease Research Group, which received research funding from Amgen. Dr. Stryker is employed by Amgen. Dr. Collins has provided consultation to Amgen, Relypsa, DaVita Clinical Research, NxStage, Keryx, and ZS Pharma.

Funding This study was supported by a research contract from Amgen Inc., Thousand Oaks, California. The contract provides for the Minneapolis Medical Research Foundation authors to have final determination of manuscript content. The authors had full control of all primary data and agree to allow the journal to review the data if asked. Dr. Sosa receives salary support from the American Heart Association Fellow to Faculty Award FTF 17400011.

\section{References}

1. Kuderer NM, Dale DC, Crawford J, Cosier LE, Lyman GH (2006) Mortality, morbidity, and cost associated with febrile neutropenia in adult cancer patients. Cancer 106:2258-2266. doi:10.1002/cncr. 21847

2. Lyman GH, Michjels SL, Reynolds MW, Barron R, Tomic KS, Yu J (2010) Risk of mortality in patients with cancer who experience febrile neutropenia. Cancer 116:5555-5563. doi:10.1002/cncr. 25332

3. Kuderer NM, Dale DC, Crawford J, Lyman GH (2007) Impact of primary prophylaxis with granulocyte colony-stimulating factor on febrile neutropenia and mortality in adult cancer patients receiving chemotherapy: a systematic review. J Clin Oncol 25:3158-3167. doi: 10.1200/JCO.2006.08.8823

4. Dale DC (2002) Colony-stimulating factors for the management of neutropenia in cancer patients. Drugs 62(Suppl 1):1-15

5. Freifeld AG, Bow EJ, Sepkowitz KA, Boeckh MJ, Ito JI, Mullen CA, Infectious Diseases Society of America et al (2011) Clinical practice guideline for the use of antimicrobial agents in neutropenic patients with cancer: 2010 update by the Infectious Diseases Society of America. Clin Infect Dis 52:e56-e93. doi:10.1093/cid/ cir073

6. Hallböök H, Lidström AK, Pauksens K (2016) Ciprofloxacin prophylaxis delays initiation of broad-spectrum antibiotic therapy and reduces the overall use of antimicrobial agents during induction therapy for acute leukemia: a single-center study. Infect Dis 48: 443-448. doi:10.3109/23744235.2016.1143963

7. Cullen M, Steven N, Billingham L, Gaunt C, Hastings M, Simmonds P et al (2005) Antibacterial prophylaxis after chemotherapy for solid tumors and lymphomas. N Engl J Med 353:099998. doi:10.1056/NEJMoa050078

8. National Comprehensive Cancer Network and NCCN Clinical practice guidelines in oncology. Myeloid growth factors. 
V.1.2014. 2014. Available at: http://www.ncen.org/professionals/ physician_gls/pdf/myeloid_growth.pdf. Accessed March 13, 2017

9. Smith TJ, Khatcheressian J, Lyman GH, Ozer H, Armitage JO, Balducci L et al (2006) 2006 update of recommendations for the use of white blood cell growth factors: an evidence-based clinical practice guideline. J Clin Oncol 24:3187-3205. doi:10.1200/JCO. 2006.06.4451

10. Vogel CL, Wojtukiewicz MZ, Carroll RR, Tjulandin SA, BarajasFigueroa LJ, Wiens BL et al (2005) First and subsequent cycle use of pegfilgrastim prevents febrile neutropenia in patients with breast cancer: a multicenter, double-blind, placebo-controlled phase III study. J Clin Oncol 23:1178-1184. doi:10.1200/JCO.2005.09.102

11. Bucaneve G, Micozzi A, Menichetti F, Martino P, Dionisi MS, Martinelli $\mathrm{G}$ et al (2005) Levofloxacin to prevent bacterial infection in patients with cancer and neutropenia. N Engl J Med 353:977987. doi:10.1056/NEJMoa044097

12. Cullen M, Steven N, Billingham L, Gaunt C, Hastings M, Simmonds P, et al (2005) Antibacterial prophylaxis after chemotherapy for solid tumors and lymphomas. N Engl J Med 2005;353: 988-998. doi: 10.1056/NEJMoa050078

13. Choi MR, Solid CA, Chia VM, Blaes AH, Page JH, Barron R et al (2014) Granulocyte colony-stimulating factor (G-CSF) patterns of use in cancer patients receiving myelosuppressive chemotherapy. Support Care Cancer 22:1619-1628. doi:10.1007/s00520-0142121-7

14. Weycker D, Sofrygin O, Seefeld K, Deeter RG, Legg J, Edelsberg J (2013) Technical evaluation of methods for identifying chemotherapy-induced febrile neutropenia in healthcare claims databases. BMC Health Serv Res 13:60. doi:10.1186/1472-6963-1360

15. von Minckwitz G, Kummel S, du Bois A, Eiermann W, Eidtmann $\mathrm{H}$, Gerber B et al (2008) Pegfilgrastim +/- ciprofloxacin for primary prophylaxis with TAC (docetaxel/doxorubicin/cyclophosphamide) chemotherapy for breast cancer. Results from the GEPARTRIO study. Ann Oncol 19:292-298. doi:10.1093/ annonc/mdm 438

16. Martin M, Lluch A, Segui MA, Ruiz A, Ramos M, Adrover E et al (2006) Toxicity and health-related quality of life in breast cancer patients receiving adjuvant docetaxel, doxorubicin, cyclophosphamide
(TAC) or 5-fluorouracil, doxorubicin and cyclophosphamide (FAC): impact of adding primary prophylactic granulocyte-colony stimulating factor to the TAC regimen. Ann Oncol 17:1205-1212. doi:10.1093/ annonc/mdl135

17. Aarts MJ, Peters FP, Mandigers CM, Dercksen MW, Stouthard JM, Nortier HJ et al (2013) Primary granulocyte colony-stimulating factor prophylaxis during the first two cycles only or throughout all chemotherapy cycles in patients with breast cancer at risk for febrile neutropenia. J Clin Oncol 31:4290-4296. doi:10.1200/JCO. 2012.44.6229

18. Cuyun CG, Barrett AM, Kaye JA, Liepa AM, Winfree KB, John WJ (2014) A comprehensive review of nongenetic prognostic and predictive factors influencing the heterogeneity of outcomes in advanced non-small-cell lung cancer. Cancer Manag Res 6:437-449. doi:10.2147/CMAR.S63603

19. Chao C, Page JH, Yang SJ, Rodriguez R, Huynh J, Chia VM (2014) History of chronic comorbidity and risk of chemotherapy-induced febrile neutropenia in cancer patients not receiving G-CSF prophylaxis. Ann Oncol 25:1821-1829. doi:10.1093/annonc/mdu203

20. Weycker D, Li X, Barron R, Wu H, Morrow PK, Xu H et al (2015) Importance of risk factors for febrile neutropenia among patients receiving chemotherapy regimens not classified as high-risk in guidelines for myeloid growth factor use. J Natl Compr Cancer Netw 13:979-986

21. National Comprehensive Cancer Network and NCCN Clinical practice guidelines in oncology. Prevention and Treatment of Cancer Related Infections. V.1.2013. 2013. Available at: http:// www.nccn.org/professionals/physician_gls/PDF/infections.pdf. Accessed March 13, 2017

22. Schwartzberg LS, Saleh M, Whittaker S, Abella E (2014) Severe neutropenia and relative dose intensity among patients $<65$ and $\geq 65$ years with non-Hodgkin's lymphoma receiving CHOP-based chemotherapy. Support Care Cancer 22:1833-1841. doi:10.1007/ s00520-014-2157-8

23. Lyman GH, Dale DC, Legg JC, Abella E, Morrow PK, Whittaker S et al (2015) Assessing patients' risk of febrile neutropenia: is there a correlation betwoon physician-assessed risk and model-predicted risk? Cancer Med 4:1153-1160. doi:10.1002/cam4.454 